ORIGINAL ARTICLE

\title{
Testing the Clinical Applicability of Resin Infiltration of Developmental Enamel Hypomineralization Lesions Using an In Vitro Model
}

\author{
Dagmar Schnabl ${ }^{1}$, Viktoria Dudasne-Orosz ${ }^{2}$, Rudolf Glueckert ${ }^{3}$, Stephan Handschuh ${ }^{4}$, Ines Kapferer-Seebacher ${ }^{5}$, \\ Herbert Dumfahrt ${ }^{6}$
}

\begin{abstract}
Aim: The aim of this study is to investigate the penetration abilities of a commercially available low-viscosity resin infiltrant into developmentally hypomineralized teeth in vitro.

Materials and methods: Four extracted third molars of a 17-year-old patient with signs of developmental enamel hypomineralization (discoloration, increased opacity, and surface roughness) were infiltrated with a low-viscosity resin mixed with a fluorescent dye, according to the manufacturer's standard protocol. Four extracted molars with sound enamel or showing only initial fissure caries were used as a control group. Specimens were embedded in polymethylmethacrylate, and grindings were prepared. High-resolution projectional radiography of the grindings was performed, and, for one specimen, quantitative micro-computed tomography was used to measure hydroxyapatite density in enamel and dentin lesions. After decalcification, the grindings were examined by reflected bright-field microscopy, wide-field fluorescence microscopy, and confocal laser scanning microscopy. Fluorescence micrographs were superimposed on the radiographs and analyzed correlatively. Results: The pattern of hypo-/demineralization in enamel and dentin in developmentally hypomineralized teeth showed a good congruence with the pattern of resin infiltration. Cavitations and dentin tubules up to a depth of $2 \mathrm{~mm}$ beyond cavitations were filled by the infiltrant. In control teeth, the penetration of the infiltrant was limited to decalcified enamel areas (initial fissure caries).
\end{abstract}

Conclusions: In vitro infiltration of developmentally hypomineralized enamel was successful.

Clinical significance: Resin infiltration might be considered as a routine procedure in the treatment of developmentally hypomineralized teeth. Further investigations with higher sample sizes, different degrees of severity, different stages of lesion extension, and modified treatment protocols are necessary.

Keywords: Cheese molars, Developmental enamel hypomineralization, Laboratory research, Low-viscosity resin, Penetration depth, Resin infiltration.

International Journal of Clinical Pediatric Dentistry (2019): 10.5005/jp-journals-10005-1609

\section{INTRODUCTION}

The technique of caries infiltration with low-viscosity resin has been conceived as a microinvasive therapy of noncavitated caries lesions in smooth tooth surfaces confined to enamel and the outer third of dentin. It affects a sealing of lesion pores, a diffusion barrier for acids and low molecular carbohydrates, an inhibition of caries progression, and a masking of white spot lesions. ${ }^{1-9}$ Fissure caries and developmental hypomineralization are about to be added to the range of clinical indications for resin infiltration. ${ }^{9-14}$

Developmental enamel hypomineralization (molar-incisor hypomineralization, $\mathrm{MIH}$; cheese molars) terms a qualitative defect of enamel with a reduced mineral content, a reduced microhardness, and an abnormality in translucency resulting in diffuse or demarcated opacities varying in color from cream to yellow or brown. ${ }^{15-17}$ Most frequently, incisors and first molars, but also other teeth, are affected..$^{18}$ Its multifactorial etiology is still unclear. ${ }^{19-21}$ The severity of the structural alteration may vary from small-area discoloration up to extensive defects with posteruptive enamel breakdown. Affected teeth are hypersensitive and prone to caries. Up to now, therapy of developmental hypomineralization has comprised prophylaxis, remineralization, desensitization, fissure sealing, restorative therapy, and tooth extraction. Infiltrant resin has been proved to penetrate into the hypomineralized enamel, however, to an unpredictable extent, pattern, and change in hardness. ${ }^{22}$
1,2,5,6 University Hospital of Dental Prosthetics and Restorative Dentistry, Medical University of Innsbruck, Innsbruck, Austria

${ }^{3}$ Department of Otorhinolaryngology, Medical University of Innsbruck, Innsbruck, Austria

${ }^{4}$ VetCore Imaging Unit, Veterinary University of Vienna, Vienna, Austria

Corresponding Author: Dagmar Schnabl, University Hospital of Dental Prosthetics and Restorative Dentistry, Medical University of Innsbruck, Innsbruck, Austria, Phone: +43 (0)512 504 27141, e-mail: dagmar.schnabl@tirol-kliniken.at

How to cite this article: Schnabl D, Dudasne-Orosz V, et al. Testing the Clinical Applicability of Resin Infiltration of Developmental Enamel Hypomineralization Lesions Using an In Vitro Model. Int J Clin Pediatr Dent 2019;12(2):126-132.

Source of support: The study was supported by the Medical University of Innsbruck

Conflict of interest: None

Infiltrant resin has been shown to penetrate into enamel that is demineralized in the course of caries, whereas sound enamel is not penetrated by the infiltrant. The aim of this investigation was to test, if infiltrant resin is capable to penetrate into developmentally hypomineralized enamel. For this purpose, in vitro resin infiltration of four extracted molars with developmental hypomineralization was 
performed and evaluated in a qualitative analysis. Four extracted molars, free from caries or displaying initial fissure caries, served as control teeth.

\section{Clinical Case Presentation}

A healthy male 17-year-old person with an uneventful medical history including the prenatal period, birth and childhood/ adolescence, and a negative family anamnesis with regard to dental abnormalities, presented four partially erupted third molars with clinical signs of developmental enamel hypomineralization (opacity, brownish, and creamy discoloration and rough surface structure affecting large parts of the occlusal and smooth tooth surfaces). All other patient's teeth showed a yellowish color, and some showed white spot lesions on smooth surfaces (Fig. 1A). The occlusal surfaces showed signs of attrition (Fig. 1B). The radiographic examination revealed initial approximal and occlusal caries lesions in upper and lower premolars and molars (Fig. 1C). Oral hygiene was in need of improvement. For orthodontic reasons, all third molars were extracted (Fig. 1D). Domestic hygiene was intensified, caries lesions within enamel or the outer third of dentin in bitewing X-rays were infiltrated (Icon; DMG, Hamburg, Germany), and more profound lesions were treated by composite restorations. The patient was provided with an occlusal splint to prevent excessive attrition during the night. A regular recall for clinical examination, professional hygiene, and eventual radiographs has been arranged.

\section{Resin Infiltration In Vitro; Materials and Methods}

\section{Specimens}

The described patient's extracted four developmentally hypomineralized third molars served as test teeth. Aside from clinical signs of developmental hypomineralization (discoloration, opacity and surface roughness), they were independently classified by two examiners as code 2 or 3 according to the International Caries
Assessment System (ICDAS). ${ }^{23}$ Four surgically removed third molars of different persons, clinically caries-free or showing initial fissure caries (classified as ICDAS code $0-1$ by the same examiners), served as control teeth. Immediately upon removal, teeth roots were cleaned by mechanical scaling and teeth were stored in distilled water.

Informed consent was obtained by the patients and/or the patients' parents prior to the use of the removed teeth for research purposes. The principles outlined in the German Ethics Committee's statement for the use of human body material in medical research were applied. ${ }^{24}$

\section{Infiltration}

The infiltrant (Icon; DMG, Hamburg, Germany) was mixed with a fluorescent dye (Lumogen Orange; BASF, Ludwigshafen, Germany) to a concentration of $0.1 \% \mathrm{w} / \mathrm{w}$ with exclusion of light as described by Belli et al. ${ }^{25}$ Tooth infiltration was performed according to the Icon application guide (standard protocol, etching with $15 \% \mathrm{HCl}$ gel for $120 \mathrm{~s}$ ). The infiltrant was light cured for $40 \mathrm{~s}$ from each direction (450 mW/cm², 420-500 nm; Polylux, Sirona Dental Sytems).

\section{Preparation for Imaging}

After infiltration, the specimens were stored in distilled water for 24 hours and then embedded in clear polymethylmethacrylate (PMMA; ProBase Clear, Ivoclar Vivadent, Schaan, Liechtenstein) in the form of cuboids.

To create slices of $500 \mu \mathrm{m}$, the blocks were cut in buccolingual direction (Band Saw 300/310 CL/CP; Exakt Apparatebau, Norderstedt, Germany). Each slice was superglued onto a microscopic slide (1733-0100 Sekundenkleber; Renfert, Hilzingen, Germany) and polished to approximately $200 \mu \mathrm{m}$ thickness under water lubrication (Micro Grinding Machine 400CS; Exakt Apparatebau and Abrasive Discs Hermes WS flex 18 B P 800, WS flex 16 P 1000 and WS flex C P 2500; Hermes Schleifmittel, Bad St. Leonhard im Lavanttal, Austria), finally rinsed with water.

One grinding per specimen showing the largest lesion extension was selected for infiltrant imaging. After the acquisition of high-resolution projectional radiographs and quantitative
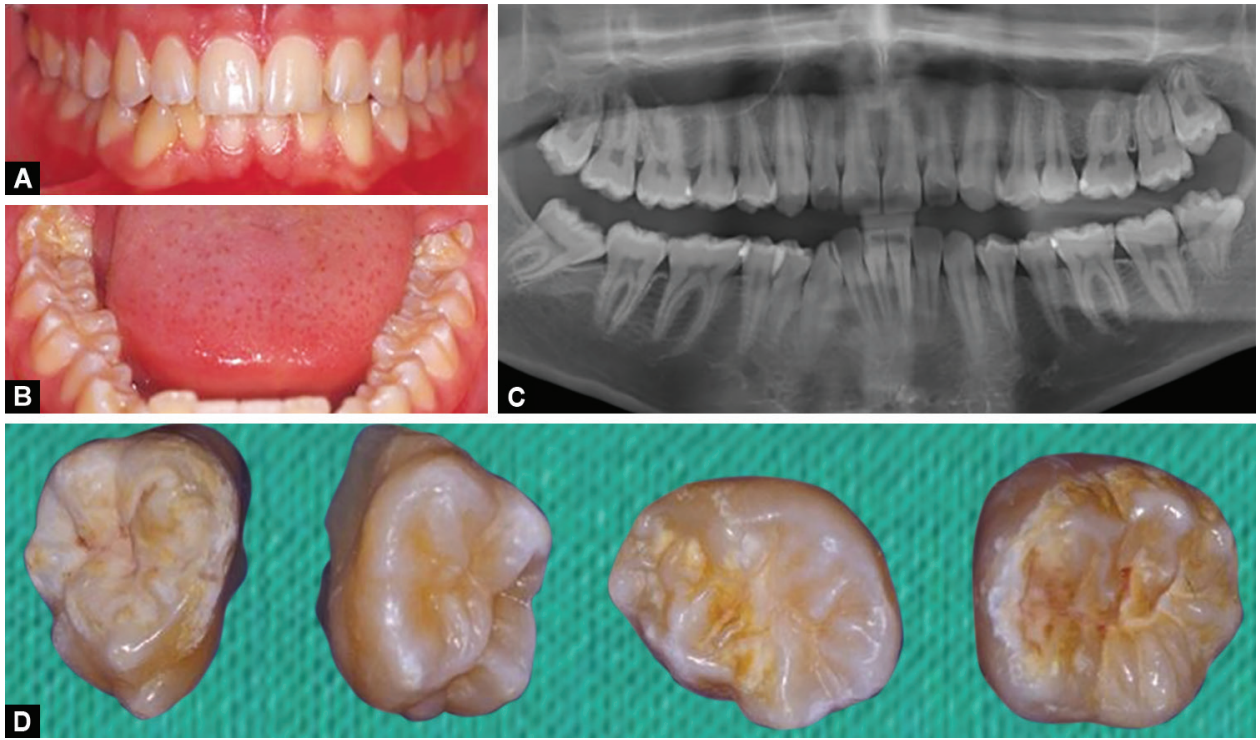

Figs 1 A to D: A 17-year-old patient's teeth with signs of developmental hypomineralization: (A) Enoral situation: yellowish teeth with white spot lesions. Oral hygiene in need of improvement; (B) Lower third molars not fully erupted. Occlusal surfaces of molars and premolars show signs of attrition; (C) Orthopantomogram: initial approximal and occlusal caries lesions in upper and lower posterior teeth; (D) Extracted test teeth (18, 28, 38 , and 48 ) with signs of hypomineralization. Note the increased opacity, dull surface, and discoloration. The teeth were subsequently affected by caries, classified as codes 2-3 according to the International Caries Detection and Assessment System (ICDAS) 

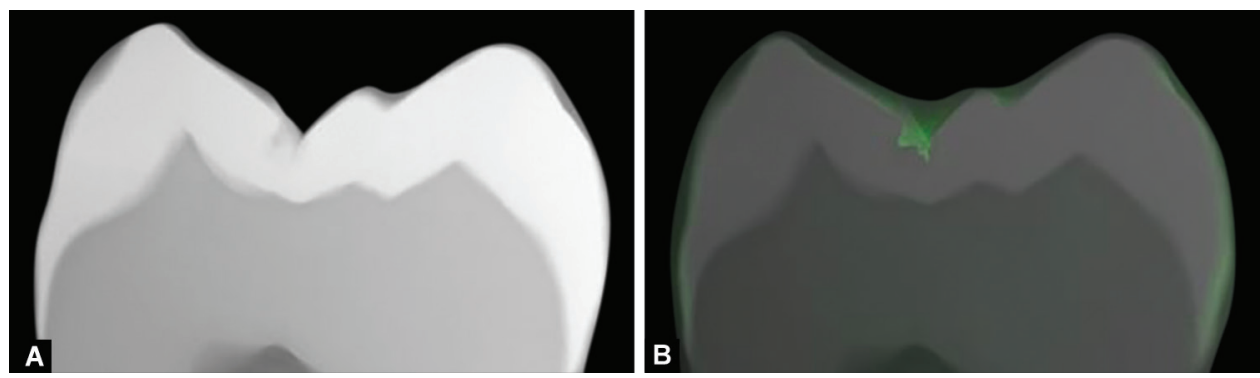

Figs 2A and B: Control tooth: (A) Radiograph shows demineralization in the outer half of enamel (E1); (B) Superimposition of radiography and fluorescence microscopy: there is a good congruence of demineralized and infiltrated areas

microcomputed tomography scans (micro-CT), the grindings were decalcified in $20 \% \mathrm{w} / \mathrm{v}$ ethylendiaminetetraacetic acid (EDTA) in phosphate buffer, $\mathrm{pH} 7.35$ at $37^{\circ} \mathrm{C}$ for $8 \mathrm{~h}$ for improved selective infiltrant imaging.

\section{High-Resolution Projectional Radiography}

High-resolution X-ray images from ground sections of molars mounted on glass slides were acquired using a microXCT-400
(Carl Zeiss X-ray Microscopy, Pleasanton, CA, USA). Glass slides were mounted vertically in a clamp sample holder. Radiographs of whole grindings were captured at $80 \mathrm{kV}$ source voltage and $100 \mu \mathrm{A}$ intensity using a $0.4 \times$ optical magnification lens. The exposure time was 30 seconds, and the voxel size was $8.91 \mu \mathrm{m}$. Detail radiographs of caries lesions were captured using a $4 \times$ optical magnification lens with identical source settings and exposure time. The voxel size of detail images was $2.24 \mu \mathrm{m}$.
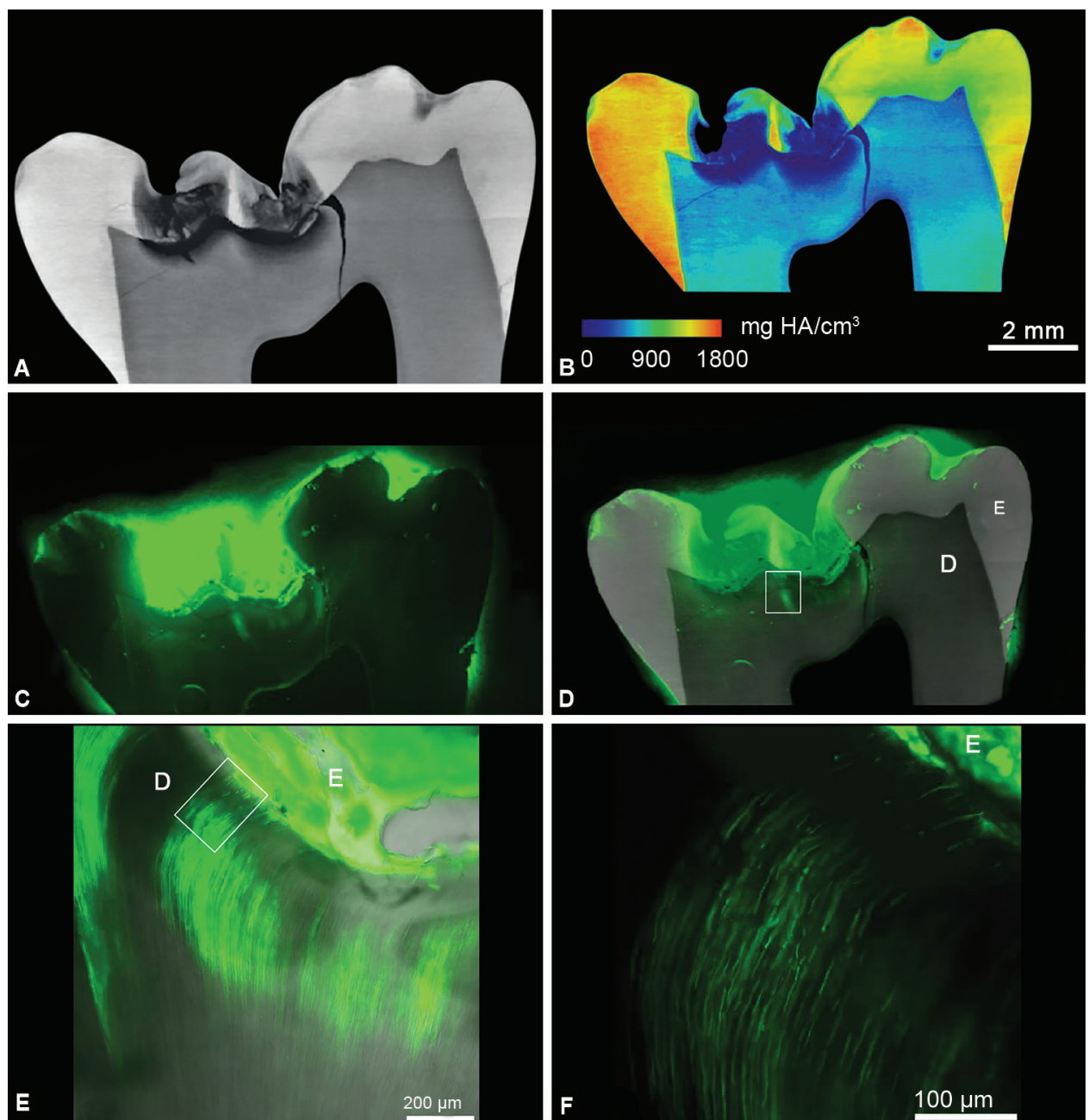

Figs 3A to F: Test tooth number 18: (A) Projectional radiograph shows enamel hypomineralization and caries with lesion extension into the intermediate third of dentin (D2); (B) Phantom-calibrated hydroxyapatite (HA) quantification visualizes enamel hypomineralization and carious decay; (C) Fluorescence microscopy reveals a consistent infiltration of hypomineralized enamel and also of decayed dentin; (D) Superimposition of images $(A)$ and $(C)$ points out congruence of patterns of hypo-/demineralization and fluorescent infiltration in enamel (E) and dentin (D); (E and F) Confocal imaging identifies the fluorescent signal within the dentin tubules penetrating far toward the pulpa 

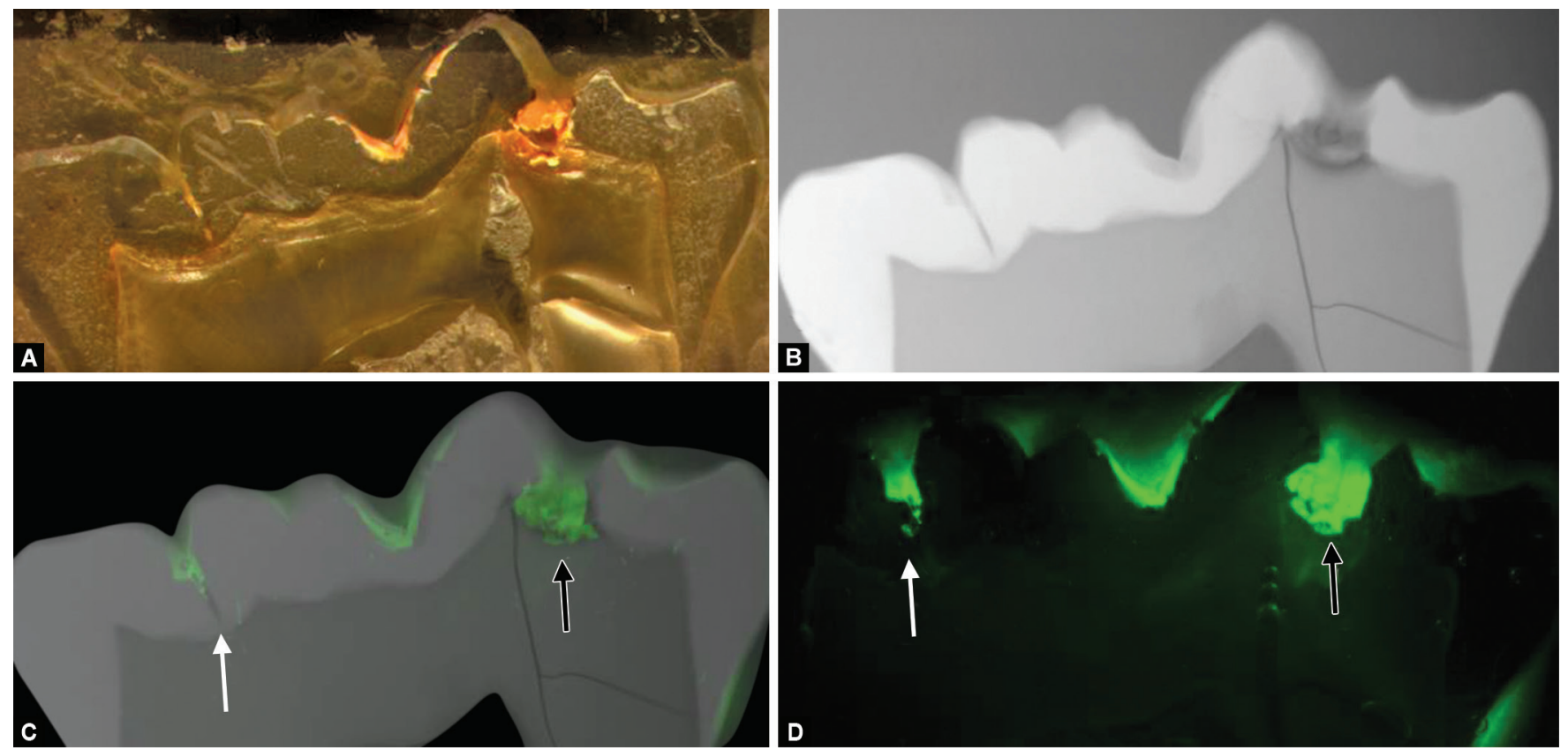

Figs 4A to D: Test tooth number 38: (A) Reflected bright-field micrograph of decalcified grinding with orange infiltrant and incomplete filling of cavitation; (B) Radiograph visualizes enamel hypomineralization and caries with lesion extension into the outer third of dentin (D1); (C) Superimposition of radiography and fluorescence microscopy shows a consistent infiltration of hypomineralized enamel and the incomplete filling of a deep fissure base (white arrow) and of an enamel/dentin cavitation (black arrow); (D) Confocal fluorescence microscopy confirms lacunas in fissure base (white arrow) and in infiltrant-filled cavitation (black arrow) and depicts a consistent infiltration of dentin around/beyond cavitation

\section{Quantitative Micro-CT}

Quantitative micro-CT was performed in one representative grinding of a test tooth. A glass slide containing the grinding was wrapped in a tissue paper and mounted vertically in a cylindrical plastic sample holder. Quantitative micro-computed tomography was performed using a Scanco Medical $\mu$ CT35 System (Brüttisellen, Switzerland) to assess mineralization of the tooth. Image acquisition was performed at $70 \mathrm{kV}$ source voltage and $114 \mu \mathrm{A}$ intensity with an angular increment of $0.18^{\circ}$ between projections and an integration time of 6 seconds per projection. CT images were reconstructed in $2048 \times 2048$ voxel matrices. The voxel resolution of reconstructed slices was $15 \mu \mathrm{m}$ (isotropic). Mineral concentrations were measured from the reconstructed slices using the hydroxyapatite-phantombased densitometry calibration of the scanner.

\section{Reflected Bright-field Microscopy and Widefield Fluorescence Microscopy}

Decalcified grindings were covered with Fluoromount- $\mathrm{G}^{\mathrm{TM}}$ (SouthernBiotech, Birmingham, AL, USA) and imaged with a calibrated upright reflected light microscope (Zeiss Lumar V12, 0.8X NeolumarS lens; Zeiss, Oberkochen, Germany) equipped a with halogen illumination and a high-pressure mercury lamp; fluorescent emission was detected with GFP filter sets (excitation bandpass 450-490 nm; emission bandpass 515-565 nm) and images acquired with a Zeiss Axiocam HRc at $3900 \times 3090$ pixel resolution using Zeiss Axiovision release 4.8. All measurements were performed with the tools of this software and images exported as.tiff files.

\section{Correlative Analysis}

High-resolution projectional radiography and wide-field fluorescence microscopy images were manually aligned using the Adobe Photoshop 6 with transparent layers and the free transform tool. By superimposition, the pattern of hypo-/demineralized areas in radiographs was correlated with the pattern of fluorescent infiltration in the corresponding microscopic images.

\section{Confocal Laser Scanning Microscopy}

Decalcified grindings were mounted with Fluoromount-G and a coverslip. Maximum fluorescent emission was interactively identified with excitation using the 405, 488, 546, and $633 \mathrm{~nm}$ laser lines and spectral detection of the emission with the Meta module of a Zeiss LSM 510 Meta (Zeiss, Oberkochen, Germany) using ZEN 2009. The 488-nm excitation proved to be most suitable, the fluorescence emission was collected from 530 to $600 \mathrm{~nm}$ and 647 to $754 \mathrm{~nm}$ at pinhole $=2$ Airy units. A Zeiss Apochromat $20 \times 0.8$ and $40 \times 1.3$ served for high-resolution scans in $1024 \times 1024$ pixels. The pixel resolution was $0.22 \mu \mathrm{m} \times$ $0.22 \mu \mathrm{m} \times 1.19 \mu \mathrm{m}$ for $40 \times$ and $0.62 \mu \mathrm{m} \times 0.62 \mu \mathrm{m} \times 7.27 \mu \mathrm{m}$ for $20 \times$. Fluorescence as well as transmission channels were recorded and digital offsets adjusted with the range indicator tool of the acquisition software.

\section{RESULTS}

\section{Extension of Hypo-/Demineralized Lesions}

High-resolution Projectional Radiography

In control teeth, any carious demineralization was confined to enamel (Fig. 2A).

Test teeth revealed extensive distinct areas of hypomineralized and/or carious enamel and extension of caries into the outer (D1) or intermediate third of the dentin (D2) (Figs 3A, 4B, 5A and B). A clear differentiation of developmentally hypomineralized from (subsequently) carious enamel is not possible: both are depicted as hypodense areas. 


\section{Quantitative Micro-CT}

In one test tooth with lesion extension D2, hypo-/demineralized enamel areas and carious dentin lesions and cavitations were assigned to the contained amount of hydroxyapatite, ranging from $1800 \mathrm{mg} / \mathrm{cm}^{3}$ in areas of apparently unaffected enamel to zero $\mathrm{mg} / \mathrm{cm}^{3}$ in cavitations by a color code (Fig. $3 \mathrm{~B}$ ).

\section{Penetration Capability of the Low-viscosity Resin}

\section{Wide-field Fluorescence Microscopy}

In control teeth, fluorescence microscopy depicted infiltrant penetration of demineralized enamel areas, whereas sound enamel showed no resin infiltration.

In test teeth, a consistent infiltration of hypomineralized and carious enamel areas by the fluorescent infiltrant, and infiltration of adjacent demineralized dentin could be proved. Even larger dentin cavitations were filled by the infiltrant, although in some cases, filling of cavitations was only partial. Nevertheless, a consistent dentin infiltration beyond and around cavitations was found (Fig. 3C).

\section{Correlative Analysis}

In control teeth, superimposition of high-resolution radiography and wide-field fluorescent light microscopy images showed congruence of the patterns of enamel demineralization and resin infiltration (Fig. 2B).

In developmentally hypomineralized test teeth, congruence of both areas of enamel hypomineralization/caries and areas of decay within dentin (displaying a reduced density in micro-CT) with the pattern of infiltration could be proved by superimposition of projectional radiography and fluorescent light microscopy images as seen in Figures 3D, 4C and 5D. Enamel and dentin cavitations were filled by the infiltrant, partly with some lacunas within the infiltrant and between the infiltrant and the enamel or dentin left blank (Fig. 4C). A consistent infiltration of dentin around and beyond cavitations could be seen. The bases of deep and shallow fissures remained partly unfilled by the fluorescent infiltrant (Fig. 4C).

\section{Confocal Laser Scanning Microscopy}

By confocal fluorescence microscopy, more detailed information about the infiltrant's abilities was obtained: a consistent infiltrant penetration of dentin tubules around and far beyond cavitations up to a depth of two millimeters could be proved (Figs 3E, F and 5E). Even most delicate side channels were filled with the infiltrant (Fig. 5F).

Confocal laser scanning microscopy also provided an improved visualization of the incomplete filling of cavitations and fissure bases (Fig. 4D).
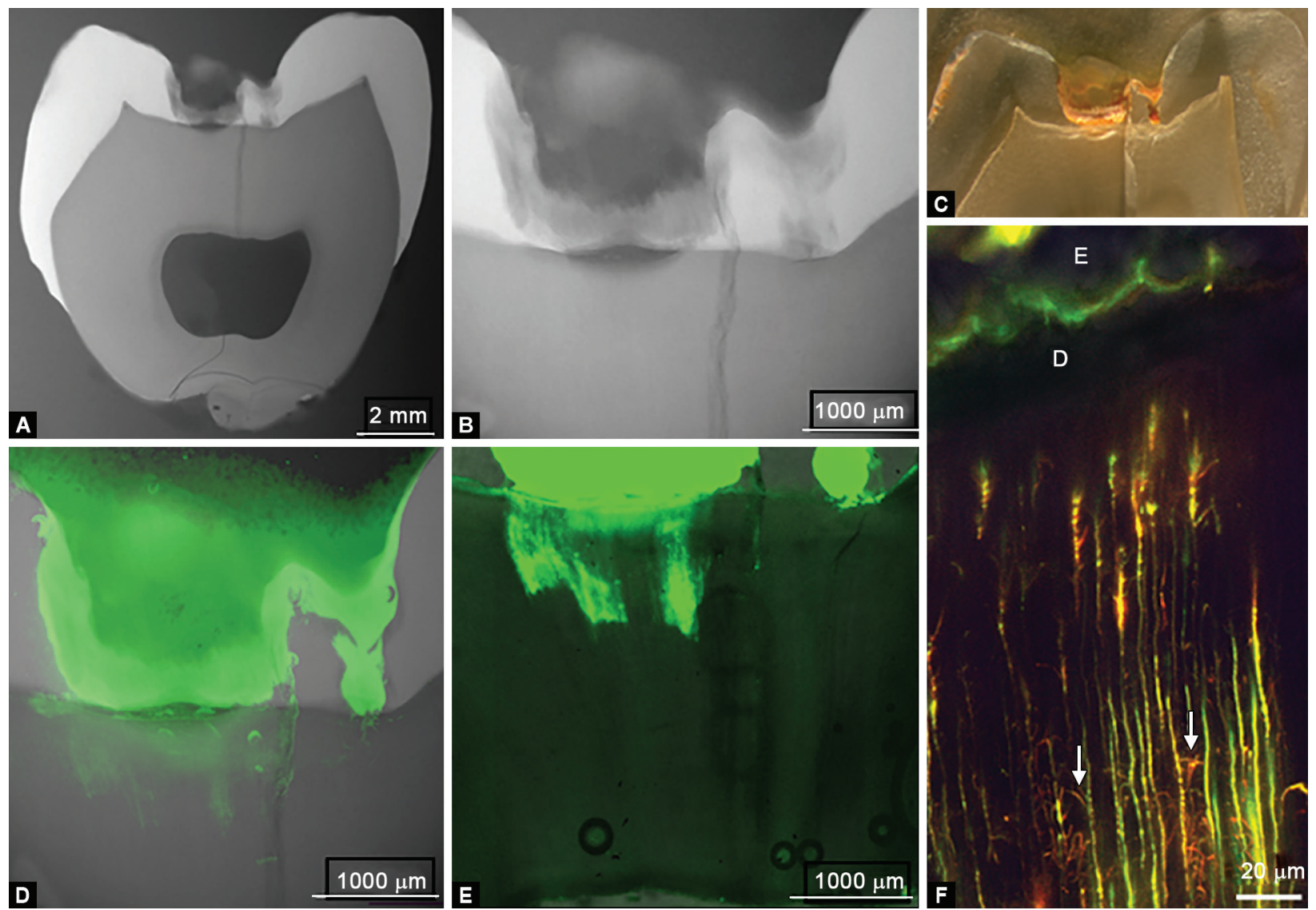

Figs 5A to F: Test tooth number 48: (A) Radiograph of the whole ground section shows lesion extension into the outer third of dentin (D1); (B) Higher magnified radiograph; (C) Reflected bright-field micrograph of the decalcified grinding visualizes infiltration of hypomineralized enamel and carious enamel and dentin; (D) Superimposition of radiography and widefield fluorescence light microscopy images reveals a consistent infiltration of hypo-/demineralized enamel and dentin, even beyond the cavitated area; (E) Confocal fluorescence microscopy better visualizes infiltration depth; (F) Maximum intensity projection of a confocal image stack with $488 \mathrm{~nm}$ excitation, red and green emission filtered detection superimposed, showing (D) dentin; penetration of the infiltrant: vertical dentin tubules and delicate horizontal side channels (arrows) are filled with the fluorescent infiltrant; (E) Enamel 


\section{Discussion}

The masking effect of infiltration therapy on fluorosis and hypoplasia stains has been described in some case reports and case series, ${ }^{12-14}$ and has been evaluated in nonrandomized studies or randomized clinical trials. ${ }^{9,26,27}$ However, little research has been published with regard to resin infiltration depth in developmentally hypomineralized enamel, and results were considered unpredictable in respect of infiltration depth and change in microhardness. ${ }^{22,28}$

In the present investigation, one extracted developmentally hypomineralized tooth was used to measure hydroxyapatite density by means of quantitative micro-CT. It showed an overall reduced content of $\mathrm{HA}$ around $1800 \mathrm{mg} / \mathrm{cm}^{3}$ in subsurface enamel, as compared to the average HA content of 2650 to $2860 \mathrm{mg} / \mathrm{cm}^{3}$ assessed in sound enamel. ${ }^{29}$

In demarcated enamel areas with a higher degree of hypomineralization, a consistent resin infiltration could be proved by use of a standard infiltration protocol. Also in control teeth, resin infiltration was limited to demineralized enamel areas (initial fissure caries). Thus, the hypothesis tested in this investigation was affirmed. The resin infiltrant's capability to penetrate into developmentally hypomineralized enamel is commensurable to its capability to penetrate into enamel demineralized by carious decay.

Resin infiltration has been conceived for noncavitated enamel lesions. Even so, under in vitro conditions, enamel and dentin cavitations of hypomineralized (and subsequently carious) teeth were filled by the infiltrant, though partly not completely. Furthermore, without any preceding dentin conditioning, the infiltrant consistently filled dentin tubules and even delicate side channels around and far beyond cavitations.

Modification of the standard infiltration protocol by an increase of etching time or a modified application of the etching agent by brushing has shown to improve infiltration depth in carious enamel lesions and fissure caries, ${ }^{11}$ and might also enhance infiltration of hypomineralized enamel. Further, oxidative pretreatment with $5.25 \%$ sodium hypochlorite plus infiltration has been proved to effect an increase in microshear bond strength in hypomineralized ename ${ }^{30}$ and may facilitate subsequent composite restorations, if necessary.

\section{Conclusion}

In this in vitro case study, developmentally hypomineralized enamel lesions were successfully infiltrated with low-viscosity resin.

Resin infiltration might be considered as a routine procedure in the treatment of developmentally hypomineralized teeth. Further investigations with higher sample sizes from different patients, with different degrees of severity and stages of lesion extension, and also the use of modified treatment protocols are needed.

\section{References}

1. Meyer-Lueckel H, Paris S. Improved resin infiltration of natural caries lesions. J Dent Res 2008 Dec;87(12):1112-1116. DOI: 10.1177/154405910808701201.

2. Kielbassa AM, Muller J, et al. Closing the gap between oral hygiene and minimally invasive dentistry: a review on the resin infiltration technique of incipient (proximal) enamel lesions. Quintessence Int 2009 Sep;40(8):663-681.

3. Kielbassa AM, Ulrich I, et al. An updated review on the resin infiltration technique of incipient proximal enamel lesions. Med Evol 2010;16(4):3-15.
4. Meyer-Lueckel H, Paris S. Infiltration of natural caries lesions with experimental resins differing in penetration coefficients and ethanol addition. Caries Res 2010 Aug;44(4):408-414. DOI: 10.1159/000318223.

5. Paris $S$, Hopfenmuller W, et al. Resin infiltration of caries lesions: an efficacy randomized trial. J Dent Res 2010 Aug;89(8):823-826. DOI: 10.1177/0022034510369289.

6. Paris $\mathrm{S}$, Meyer-Lueckel H. Inhibition of caries progression by resin infiltration in situ. Caries Res 2010 Jan;44(1):47-54. DOI: 10.1159/000275917.

7. Paris S, Schwendicke F, et al. Masking of white spot lesions by resin infiltration in vitro. J Dent 2013 Nov;41(5):e28-e34. DOI: 10.1016/ j.jdent.2013.04.003.

8. Knösel M, Eckstein A, et al. Durability of esthetic improvement following Icon resin infiltration of multibracket-induced white spot lesions compared with no therapy over 6 months: a single-center, split-mouth, randomized clinical trial. Am J Orthod Dentofacial Orthop 2013 Jul;144(1):86-96. DOI: 10.1016/j.ajodo.2013.02.029.

9. Borges $A B$, Caneppele TM, et al. Is resin infiltration an effective esthetic treatment for enamel development defects and white spot lesions? A systematic review. J Dent 2017 Jan;56:11-18. DOI: 10.1016/j. jdent.2016.10.010.

10. Paris S, Lausch J, et al. Comparison of sealant and infiltrant penetration into pit and fissure caries lesions in vitro. J Dent 2014 Apr;42(4):432438. DOI: 10.1016/j.jdent.2014.01.006.

11. Lausch J, Paris S, et al. Resin infiltration of fissure caries with various techniques of pretreatment in vitro. Caries Res 2015;49(1):50-55. DOI: 10.1159/000366082.

12. Muñoz MA, Arana-Gordillo LA, et al. Alternative esthetic management of fluorosis and hypoplasia stains: blending effect obtained with resin infiltration techniques. J Esthet Restor Dent 2013 Feb;25(1):32-39. DOI: 10.1111/j.1708-8240.2012.00527.x.

13. Torres $C R$, Borges $A B$. Color masking of developmental enamel defects: a case series. Oper Dent 2015 Jan-Feb;40(1):25-33. DOI: 10.2341/13-346-T.

14. Wang $Y$, Sa $Y$, et al. Minimally invasive treatment for esthetic management of severe dental fluorosis: a case report. Oper Dent 2013 Jul-Aug;38(4):358-362. DOI: 10.2341/12-238-S.

15. Contaldo $M, D i$ Stasio $D$, et al. Non-invasive in vivo visualization of enamel defects by reflectance confocal microscopy (RCM). Odontology 2015 May;103(2):177-184. DOI: 10.1007/s10266-014-01554.

16. Crombie FA, Manton DJ, et al. Characterisation of developmentally hypomineralised human enamel. J Dent 2013 Jul;41(7):611-618. DOI: 10.1016/j.jdent.2013.05.002.

17. Jälevik B, Norén JG. Enamel hypomineralization of permanent first molars: a morphological study and survey of possible aetiological factors. Int J Paediatr Dent 2000 Dec;10(4):278-289. DOI: 10.1046/j.1365-263x.2000.00210.x.

18. Lygidakis NA, Wong F, et al. Best Clinical Practice Guidance for clinicians dealing with children presenting with Molar-IncisorHypomineralisation (MIH): An EAPD Policy Document. Eur Arch Paediatr Dent 2010 Apr;11(2):75-81. DOI: 10.1007/BF03262716.

19. Crombie F, Manton D, et al. Aetiology of molar-incisor hypomineralization: a critical review. Int J Paediatr Dent 2009 Mar;19(2):73-83. DOI: 10.1111/j.1365-263X.2008.00966.x.

20. Garg N, Jain AK, et al. Essentiality of early diagnosis of molar incisor hypomineralization in children and review of its clinical presentation, etiology and management. Int J Clin Pediatr Dent 2012 Sep;5(3):190196. DOI: 10.5005/jp-journals-10005-1164.

21. Salanitri S, Seow WK. Developmental enamel defects in the primary dentition: aetiology and clinical management. Aust Dent J 2013 Jun;58(2):133-140. DOI: 10.1111/adj.12039.

22. Crombie F, Manton D, et al. Resin infiltration of developmentally hypomineralised enamel. Int J Paediatr Dent 2014 Jan;24(1):51-55. DOI: 10.1111/ipd.12025.

23. Pitts NB, Ekstrand KR. ICDAS Foundation: International Caries Detection and Assessment System (ICDAS) and its International 
Caries Classification and Management System (ICCMS) - methods for staging of the caries process and enabling dentists to manage caries. Community Dent Oral Epidemiol 2013 Feb;41(1):e41-e52. DOI: 10.1111/ cdoe.12025.

24. http://www.zentrale-ethikkommission.de/page.asp?his=0.1.21.

25. Belli $R$, Rahiotis $C$, et al. Wear and morphyology of infiltrated white spot lesions. J Dent 2011 May;39(5):376-385. DOI: 10.1016/ j.jdent.2011.02.009.

26. Kim S, Kim EY, et al. The evaluation of resin infiltration for masking labial enamel white spot lesions. Int J Paediatr Dent 2011 Jul;21(4): 241-248. DOI: 10.1111/j.1365-263X.2011.01126.x.

27. Gugnani N, Pandit l, et al. Comparative evaluation of esthetic changes in nonpitted fluorosis stains when treated with resin infiltration, in-office bleaching, and combination therapies. J Esthet Restor Dent 2017 Sep;29(5):317-324. DOI: 10.1111/jerd.12312.

28. Kumar H, Palamara JEH, et al. An investigation into the effect of a resin infiltrant on the micromechanical properties of hypomineralized enamel. Int JPaediatr Dent 2017 Sep;27(5):399-411.DOI: 10.1111/ipd.12272.

29. Anderson P, Elliott JC, et al. A comparison of the mineral content of enamel and dentine in human premolars and enamel pearls measured by X-ray microtomography. Arch Oral Biol 1996 Mar; 41(3):281-290. DOI: 10.1017/0003-9969(95)00122-0.

30. Chay PL, Manton D, et al. The effect of resin infiltration and oxidative pre-treatment on microshear bond strength of resin composite to hypomineralised enamel. Int J Paediatr Dent 2014 Jul;24(4):252-267. DOI: 10.1111/ipd.12069. 